\title{
INICIAÇÃO CIENTÍFICA JÚNIOR COMO MOTIVAÇÃO PARA INSERÇÃO NO ENSINO SUPERIOR
}

Myllena G. da Silva - myygarcia@ gmail.com

Universidade Católica Dom Bosco, Engenharia de Computação

Av. Tamandaré, 6000

79117-900 - Campo Grande - Mato Grosso do Sul

Naji R. N. Ama - naji@usp.br

Universidade Católica Dom Bosco, Engenharia de Controle e Automação

Av. Tamandaré, 6000

79117-900 - Campo Grande - Mato Grosso do Sul

Mauro C. Pereira - maurocp@gmail.com

Instituto Federal do Mato Grosso do Sul, Engenharia Elétrica

R. Taquarí, 831

79100-510 - Campo Grande - Mato Grosso do Sul

Resumo: Este artigo apresenta a importância do incentivo à ciência e tecnologia para os estudantes do ensino médio de escolas públicas. Sendo assim, foi desenvolvido durante um periodo de seis meses, um robô jogador de futebol da categoria IEEE-VSSS (Very Small Size Soccer) conforme suas regras oficiais, junto aos alunos participantes do PIBIC Júnior (Programa Institucional de Bolsas de Iniciação Científica - Júnior) vinculados à UCDB (Universidade Católica Dom Bosco). O objetivo é promover o interesse dos estudantes do ensino médio em relação a programação, conceitos básicos de física e eletrônica e princípios de robótica como meio de motivação na inserção do ensino superior para a formação de futuros engenheiros.

Palavras-chave: Educação. Robótica. Tecnologia. Ensino Médio.

\section{INTRODUÇÃO}

$\mathrm{Na}$ rede pública, o percentual de estudantes que completaram o ensino médio e ingressaram no ensino superior em 2018 é de 35,9\%, de acordo com os dados da Síntese de Indicadores Sociais divulgados pelo Instituto Brasileiro de Geografia e Estatística (IBGE). Além disso, há uma deficiência nas disciplinas que envolvem áreas de programação e robótica que são fundamentais para o mercado de exatas.

Com o intuito de fortalecer o processo de disseminação de conhecimento científico e tecnológicos básicos, o Programa Institucional de Bolsas de Iniciação Científica - Júnior, PIBIC Júnior, é fundamental para a convivência dos alunos da rede pública dentro do meio acadêmico, onde terão a experiência de aprender ciência e tecnologia, além de desenvolver projetos dentro da área de engenharia.

O IEEE Very Small Size Soccer (VSSS) é uma categoria de competição de futebol de robôs que pode ser encontrado em competições como: Competição Brasileira de Robótica 
(CBR) e Competição Latino-Americana de Robótica (LARC). Essa proposta estimula o desenvolvimento de lógica, tecnologias e habilidades, tanto individuais quanto em equipe, que são aproveitadas em futuras experiências no mercado de trabalho.

Sendo assim, este trabalho aborda o projeto da Universidade Católica Dom Bosco (UCDB) com o Programa Institucional de Bolsas de Iniciação Científica - Júnior (PIBICJúnior) que oferece aos estudantes um ambiente onde possam aprender o processo de trabalhos científicos, como a construção de um robô jogador de futebol, proposto no plano de pesquisa, junto com um professor orientador e com acadêmicos da instituição, por meio de aulas semanais. Durante o período dessas aulas, é ensinado conteúdos de eletrônica, física e linguagem de programação Arduíno, com o auxílio de softwares de modelagem e a IDE do Arduíno.

\section{METODOLOGIA}

Como forma de tornar acessível o estudo de robótica para alunos entre 15 e 17 anos, foi proposto uma metodologia que adapte tanto os estudantes quanto os acadêmicos da instituição de ensino UCDB. Para isso, foi criado um ambiente online e compartilhado, no Google Drive, onde ficaram disponíveis cronogramas das atividades, relatórios semanais e materiais didáticos de ensino da área específica. Além disso, foram usados softwares de apoio como ferramentas para o aprendizado e componentes eletrônicos para o manuseio e implementação das atividades propostas.

\subsection{Cronograma de atividades}

Por meio planilhas, os acadêmicos da UCDB e o professor orientador deste projeto registraram, de maneira padronizada, a data e o conteúdo ministrado, como apresenta a Tabela 1. Dessa forma manteve organizado e salvos o histórico de todo o ciclo do projeto.

Tabela 1 - Cronograma de Atividades

\begin{tabular}{|l|l|}
\hline Data & Atividades \\
\hline $07 / 08 / 2019$ & Primeiro encontro; apresentação do lab; montagem do lattes; assinatura do termo \\
\hline & Plano de pesquisa. \\
\hline Cadastro de usuário e senha; \\
\hline $14 / 08 / 2019$ & Apresentação e estabelecimento dos planos de pesquisa; \\
\hline & Apresentação do Arduino e sua programação (Básico -> Blink Led); \\
\hline & Apresentação do simulador do Arduino Tinkerçad \\
\hline & Assinatura dos termos de compromisso dos voluntários; \\
\hline & Credenciamento dos alunos para usuários e senhas; \\
\hline
\end{tabular}

Fonte: Elaboração própria, 2019.

\subsection{Relatórios semanais}

Os relatórios semanais consistem em documentar as atividades realizadas pelos alunos da equipe do PIBIC-Júnior. Portanto, cada aluno produziu seu próprio relatório digitalmente, onde foi comentado os experimentos realizados durante o dia, como apresentado na Figura 1. Esses relatórios tem como finalidade a análise de desempenho do aprendizado dos alunos.

Figura 1 - Relatório semanal 


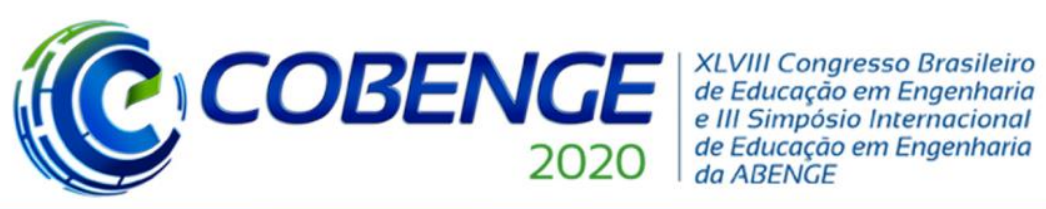

"Os desafios para formar hoje o engenheiro do amanhã"

\begin{abstract}
$14 / 08 / 2019$
Hoje vimos como será o nosso projeto de pesquisa, o que vamos aprender durante o projeto de robótica e a divisão dos estudos apresentados. Vimos também sobre VSSS com os campeonatos de futebol, junto com a construção do robo para o jogo, demos uma leve olhada sobre desenhos em 3D e como construí-los e para a finalização brincando um pouco com o protótipo do professor.
\end{abstract}

\title{
$21 / 08 / 2019$
}

Vimos hoje sobre o arduino e suas composições, como funciona e suas peças; vimos também um site ( Tinkercad), onde podemos montar diversos trabalhos antes de realmente testar no arduino real e lemos algumas coisa nas nossas apostilas.

Fonte: Equipe do PIBIC-Júnior, 2019.

\subsection{Materiais didáticos}

De maneira a impulsionar o processo de ensino e aprendizagem (SOUSA, 2015), os materiais didáticos auxiliaram tanto os acadêmicos quanto os estudantes do ensino médio durante o ciclo do projeto. Foi planejado ministrar um conteúdo específico semanalmente, cada qual com um material para facilitar o entendimento e a explicação da matéria, que ficaram disponíveis no ambiente compartilhado para acesso de todos a qualquer momento.

\subsection{Softwares e componentes eletrônicos}

A robótica abrange temas variados desde lógica, programação e eletrônica. Portanto, para esse projeto, foram utilizados softwares que serviram como ferramentas de auxílio para o aprendizado dos estudantes. Esses softwares são: Tinkercad, MIT App Inventor, FreeCAD e a IDE Arduino. Além dos softwares, também foi utilizado protoboards com componentes eletrônicos para a construção físicas de circuitos

\section{Tinkercad}

O Tinkercad é um programa de modelagem 3D de acesso gratuito e online, disponível em: https://www.tinkercad.com/. Tem como funcionalidade a criação de circuitos analógicos e digitais de maneira simples e fácil, como apresenta a Figura 2, além de projetar objetos 3D e desenvolver blocos de códigos

Figura 2 - Simulação no Tinkercad

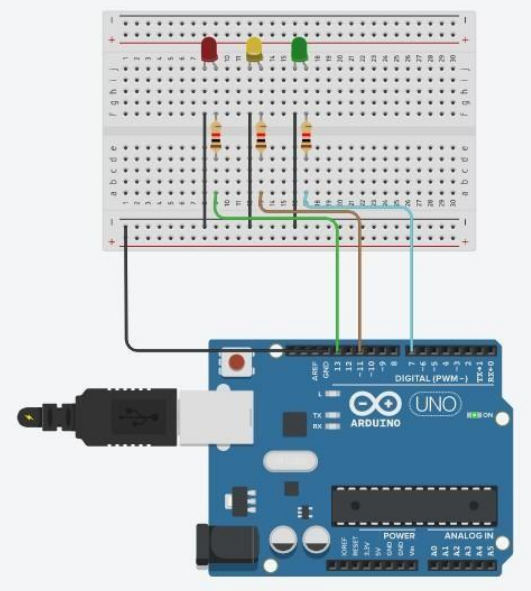

Fonte: Equipe do PIBIC-Júnior, 2019. 
(C) COBENGE

"Os desafios para formar hoje o engenheiro do amanhã"
O1 a 03 de dezembro Evento On-line

\section{MIT App Inventor}

Originalmente criado pela Google e mantido pelo Massachussetts Institute of Technology (MIT), o App Inventor é uma aplicação de código aberto à fim de criar um ambiente simplificado para que pessoas com pouco conhecimento em programação possa criar aplicativos para o sistema operacional Android, disponível em: https://appinventor.mit.edu/. Essa ferramenta foi utilizada neste projeto como forma de introdução à programação. Sendo assim, foi ministrado o passo a passo do desenvolvimento de uma aplicação simples para os estudantes utilizando bloco de códigos, conforme a Figura 3.

Figura 3 - Bloco de código no MIT App Inventor
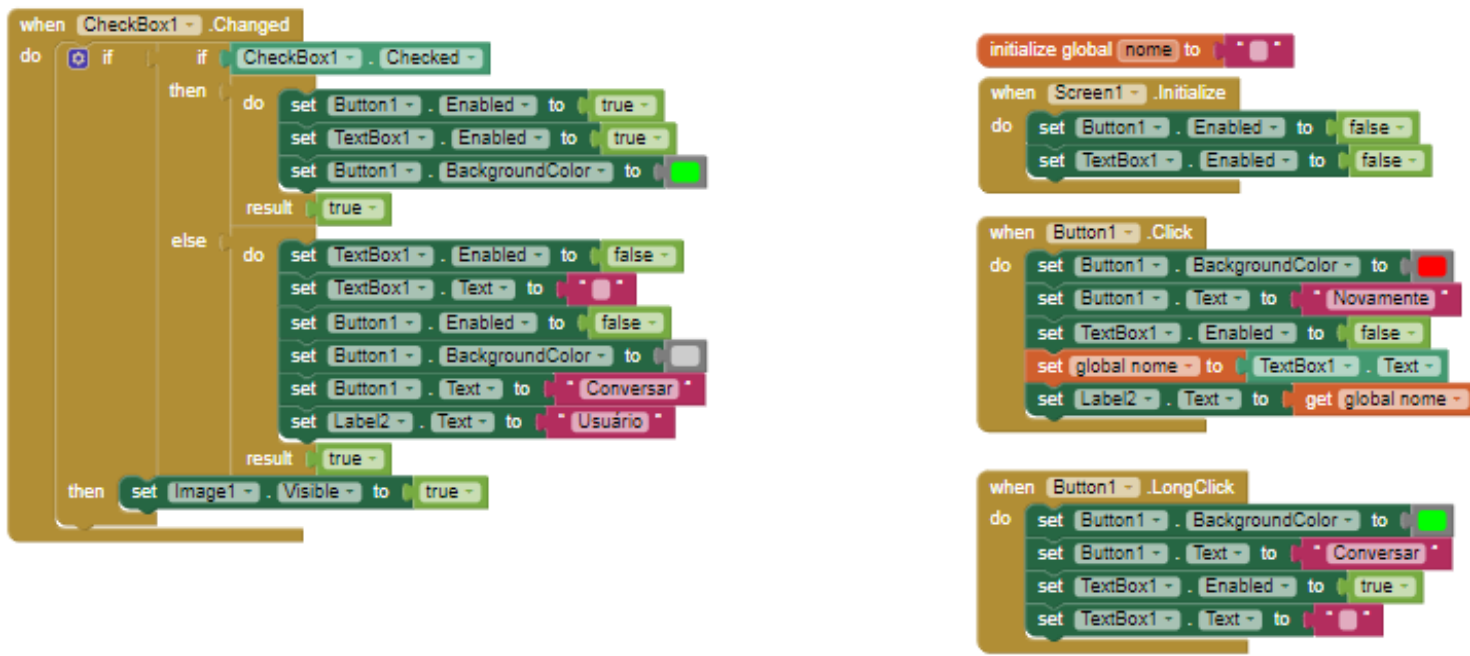

Fonte: Equipe do PIBIC-Júnior, 2019.

O MIT App Inventor também integra a interface gráfica na sua aplicação, como mostra a Figura 4, que torna o aprendizado da programação mais fácil e acessível.

Figura 4 - Interface da aplicação no MIT App Inventor

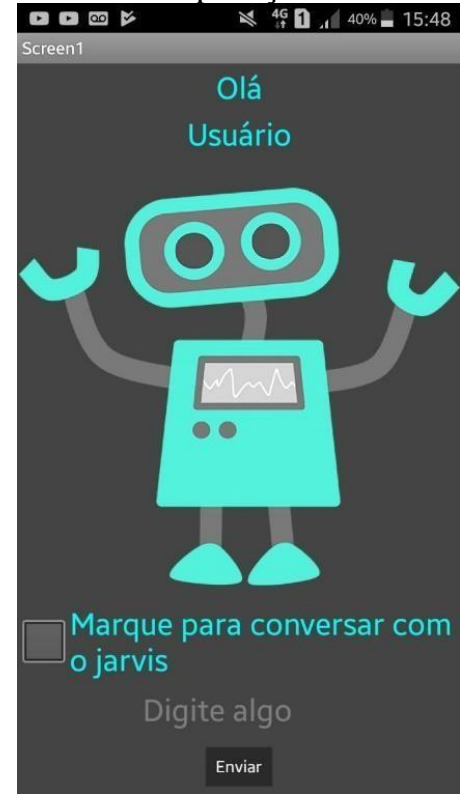

Fonte: Equipe do PIBIC-Júnior, 2019. 


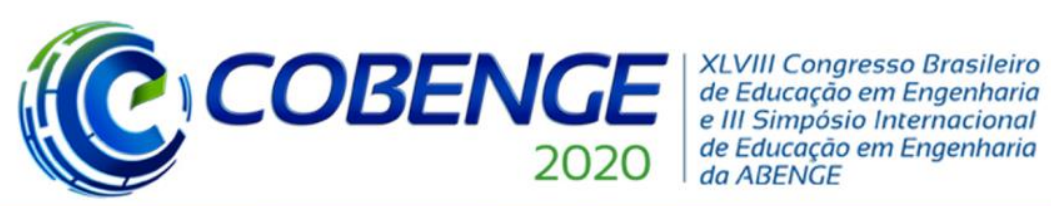

"Os desafios para formar hoje o engenheiro do amanhã"
$\mathrm{O}$ a $\mathrm{O3}$ de dezembro Evento On-line

\section{FreeCAD}

O FreeCAD é um modelador 3D de código aberto voltado diretamente para o uso em engenharia mecânica e design de produto, contudo abrangendo em outros ramos das engenharias e arquitetura, disponível em: https://www.freecadweb.org/. Além de poder criar objetos de maneira paramétrica, esse software disponibiliza recursos como: ferramentas de Análise de Elementos Finitos (FEA), Geodata e um módulo de simulação de robô que estuda seus movimentos. A finalidade de utilizar o FreeCAD neste projeto é modelar a estrutura física do robô de acordo com o plano de pesquisa. Cada estudante modelou individualmente sua estrutura seguindo as medidas dirigidas pelo professor e acadêmicos, conforme a Figura 5.

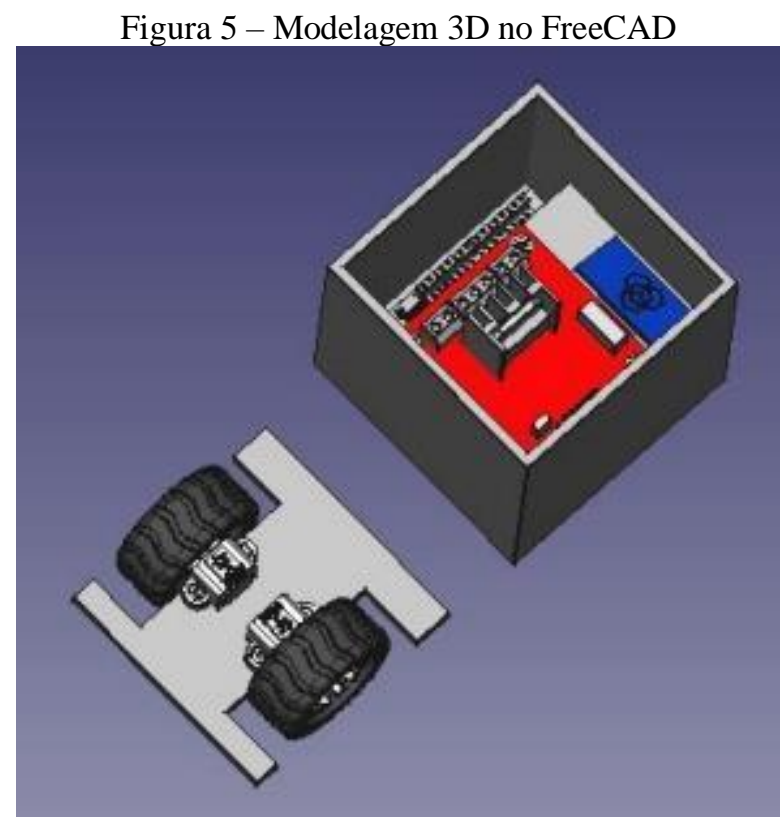

Fonte: Equipe do PIBIC-Júnior, 2019

\section{Arduino}

Ao longo dos anos, o Arduino, plataforma eletrônica de código aberto, tem sido utilizado em diversos projetos tanto do cotidiano quanto científicos. Essa plataforma oferece softwares e hardwares que são utilizadas no meio acadêmico por se tratar de uma ferramenta prática, disponível em: https://www.arduino.cc/en/Main/Software. Neste projeto, todos os conteúdos que exigiam eletrônica foi baseado no uso do Arduino Uno, apresentado na Figura 6.

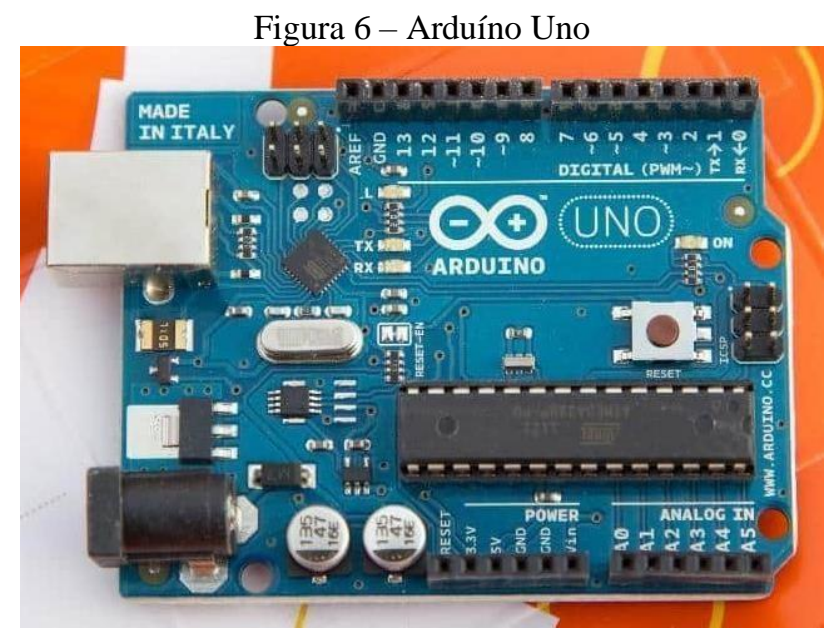

Fonte: Fábio Souza, 2013. 
(C) COBENGE

"Os desafios para formar hoje o engenheiro do amanhã"
$\mathrm{Ol}$ a $\mathrm{O} 3$ de dezembro Evento On-line

\section{RESULTADOS}

O tempo total deste projeto foi de treze semanas. Os experimentos e conteúdos montados e apresentados para os estudantes foram distribuídos de maneira gradual. Alguns experimentos foram mais longos e precisaram de mais tempo para realizar. A Figura 7 mostra o percentual dos conteúdos dos experimentos em relação ao tempo total do projeto.

Figura 7 - Distribuição dos experimentos durante o tempo total do projeto (treze semanas)

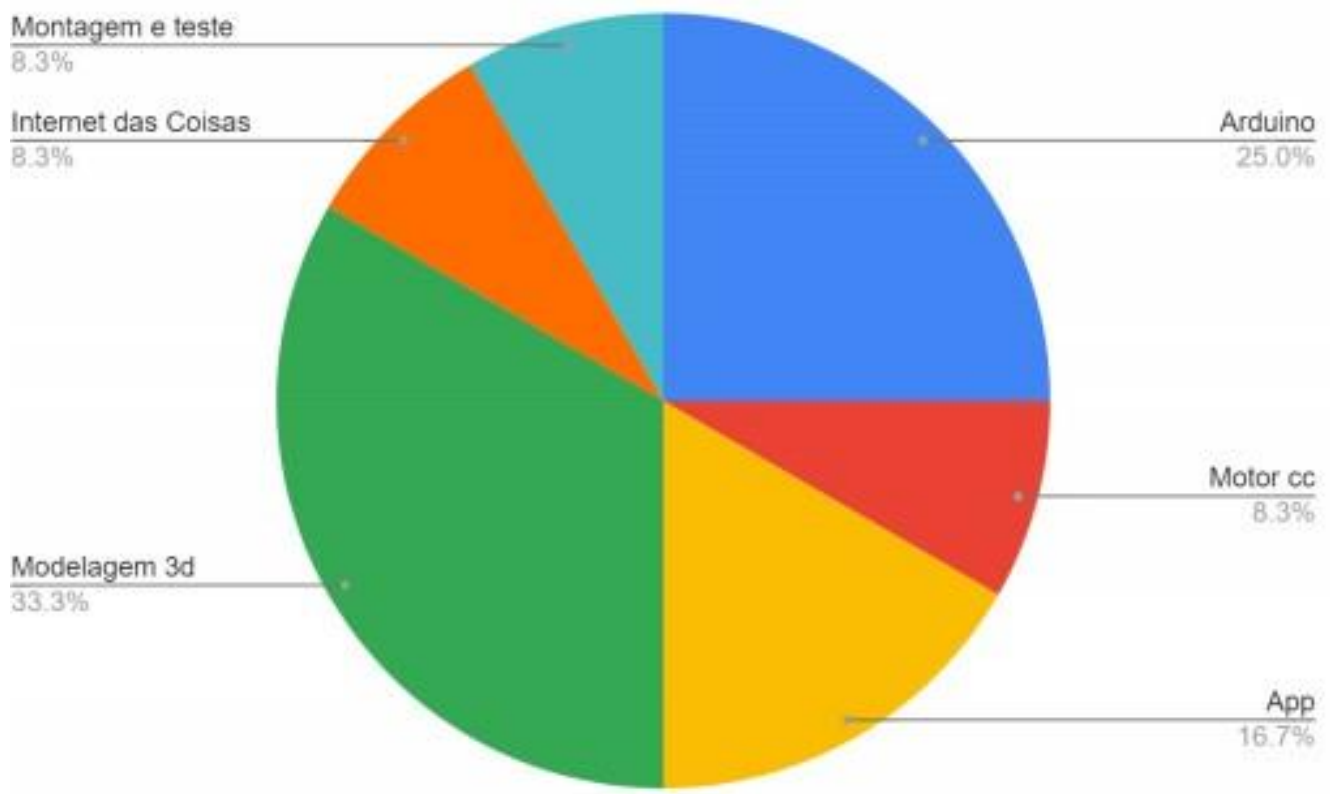

Fonte: Elaboração própria, 2019.

As três primeiras semanas de aula começaram com a demonstração do plano de pesquisa para o projeto assim como sua divisão. Além disso, foi mostrado aos alunos um protótipo para se familiarizarem e foram introduzidos ao Arduino, com aula teórica e prática, tanto com hardware e software com LED RGB para um semáforo, como mostra a Figura 2. A Figura 8 apresenta a implementação de um circuito com LED RGB e sua programação, feitas pelo estudante Pedro do da equipe do PIBIC-Júnior utilizando Arduino.

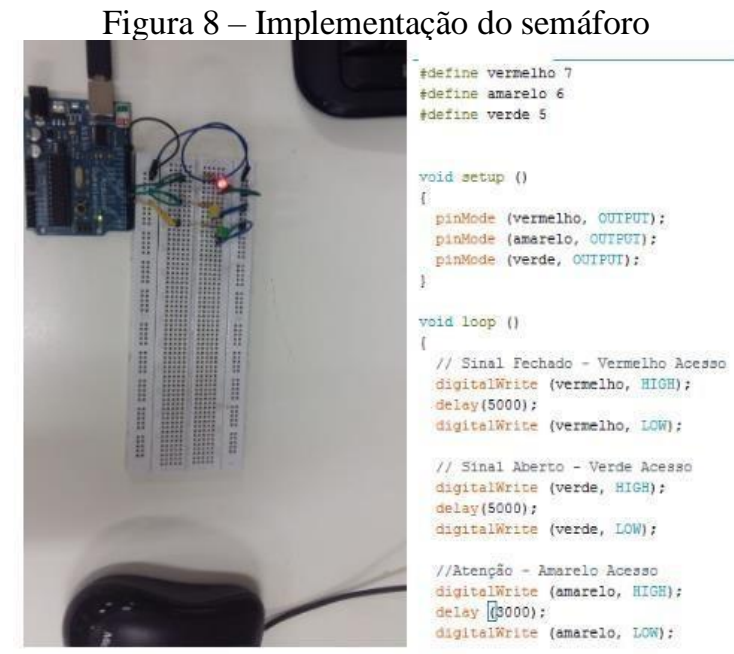

Fonte: Equipe do PIBIC-Júnior, 2019. 
Enquanto isso, nas quarta e quinta semanas, os estudantes foram introduzidos aos conteúdos de potenciômetro a partir do funcionamento de um carro já montado com Arduino e motor de corrente contínua. A aula teve como propósito obter os resultados do circuito por meio do osciloscópio. No entanto, houve dificuldade para o entendimento desses conteúdos. Os questionamentos foram esclarecidos com o passar do tempo com a ajuda dos acadêmicos e do professor orientador. A Figura 9 apresenta o gráfico do circuito com potenciômetro obtido pela Giovana da equipe do PIBIC-Júnior como resultado após o experimento proposto.

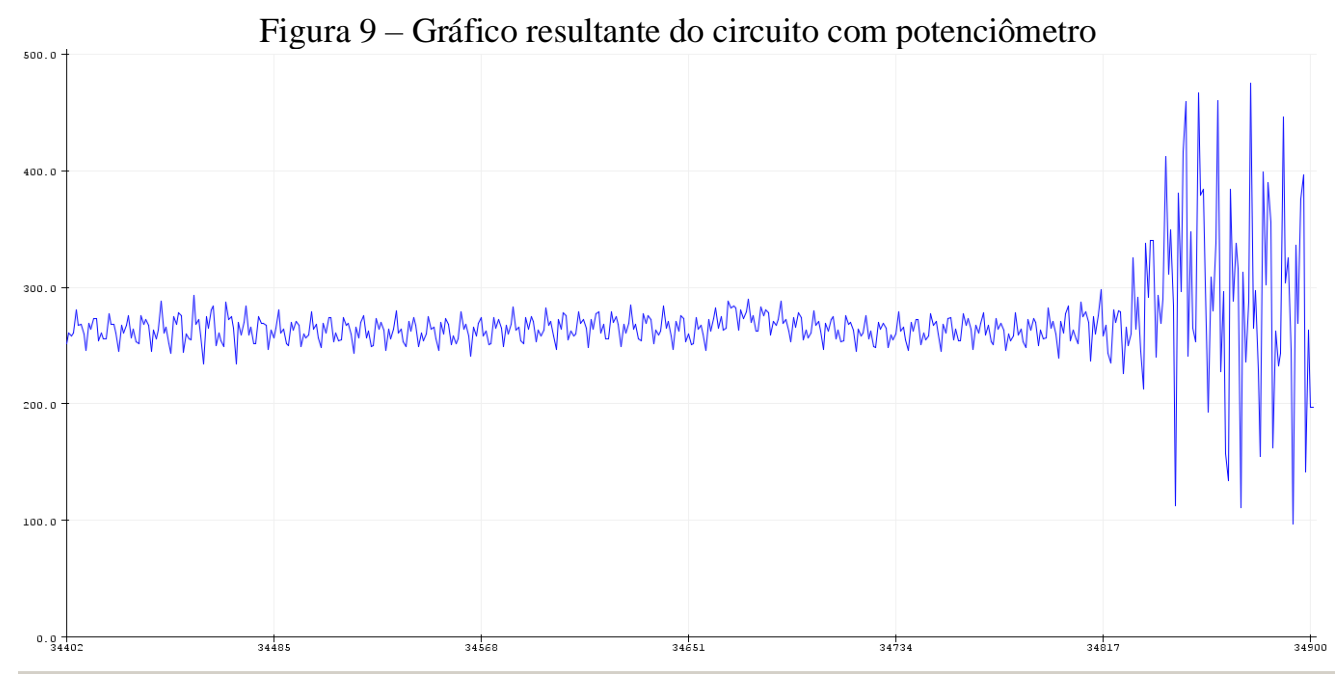

Fonte: Equipe do PIBIC-Júnior, 2019.

O funcionamento do carro apresentado aos alunos necessitava de uma ponte $\mathrm{H}$. Apesar de se tratar de um componente novo, não houve dificuldade por parte dos estudantes para o entendimento. A partir dessa aula foi possível perceber a evolução dos estudantes diante do acompanhamento oferecido.

Da sexta semana em diante foi focado no desenvolvimento do robô proposto no plano de pesquisa, da categoria IEEE-VSSS. Contudo, as regras oficiais exigem o funcionamento do robô por meio de visão computacional. Por se tratar de um conteúdo muito extenso para estudantes do ensino médio, a proposta foi alterada para o controle por aplicativo via Bluetooth. Sendo assim, foi introduzido os conceitos de criação de aplicativos usando o programa MIT App Inventor. As Figuras 3 e 4 mostram os resultados obtidos durante essa aula cujo foram satisfatórios.

Já nas aulas de modelagem 3D, utilizando o programa FreeCAD, a concepção da estrutura do robô jogador de futebol estava mais clara, como mostra a Figura 5. Enquanto na penúltima semana, na introdução sobre Internet das Coisas, houve mais entusiasmo por parte dos estudantes, assim como na última semana, quando foi feita a montagem do robô usando MDF.

\section{CONSIDERAÇÕES FINAIS}

Neste artigo foi apresentado o projeto da Universidade Católica Dom Bosco junto com o PIBIC-Júnior que visa a inclusão de estudantes da rede pública no meio acadêmico, por meio de aulas com experimentos práticos e teóricos e com a convivência de acadêmicos universitários.

Durante este projeto foi possível observar a evolução dos estudantes do ensino médio perante as disciplinas ministradas. Inicialmente os alunos não tinham nenhum ou pouco 
conhecimento de robótica, programação, eletrônica e física. Ao decorrer do projeto, demonstraram dominar os conceitos básicos proposto a eles. Os experimentos realizados são os básicos da área de engenharia, que quando introduzido aos jovens de maneira dinâmica, os motivam para o ingresso na área de exatas. A convivência no meio acadêmico desperta o interesse dos jovens para sua inserção em universidades, como também influencia a buscar conhecimentos científicos além de formar futuros engenheiros.

\title{
REFERÊNCIAS
}

FREITAS, Adriel O. de.; ANDRADE, Murilo M. X.; ALMEIDA, Rômulo N. C. de. Iniciativa de Ensino de Lógica e Microcontroladores para Alunos do Ensino Médio. In: XLVII Congresso Brasileiro de Educação em Engenharia e II Simpósio Internacional de Educação em Engenharia da ABENGE, 2019, Fortaleza. Anais. Sobral, 2019.

\section{LATIN AMERICAN ROBOTICS COMPETITION. IEEE Very Small Size}

Soccer (VSSS). Disponível em: http://www.cbrobotica.org/?page_id=81. Acesso em: 01 mai. 2020.

PIBIC Junior. Disponível em: http://www.fundect.ms.gov.br/programas/pibic- junior/. Acesso em: 01 mai. 2020.

SOUSA, Rayssa Kathleen R. de. Reflexões sobre os materiais didáticos: Qual a relação entre os professores e esses recursos em sala de aula?. In: II Congresso Nacional de Educação, 2015, Campina Grande. Anais. 2015.

SOUZA, Fábio. Arduino UNO. Disponível em: https://www.embarcados.com.br/arduinouno/. Acesso em: 24 set. 2020.

VALOR ONLINE. Estudante de escola paga tem o dobro da chance de entrar na faculdade. Disponível em: https://g1.globo.com/educacao/noticia/2018/12/05/estudante-deescola-paga-tem- o-dobro-da- chance-de-entrar-na-faculdade-aponta-estudo-do-ibge.ghtml. Acesso em: 30 abr. 2020.

\section{JUNIOR SCIENTIFIC INICIATION AS MOTIVATION FOR INSERTION IN HIGHER EDUCATION}

\begin{abstract}
This article presents the importance of encouraging science and technology for high school students in public schools. To this end, a robot football player of the IEEE-VSSS (Very Small Size Soccer) category was developed over a period of six months, according to its official rules, with the students participating in the PIBIC Junior (Institutional Program for Scientific Initiation Scholarships - Junior) linked to UCDB (Universidade Católica Dom Bosco). The objective is to promote the interest of high school students in relation to programming, basic concepts of physics and electronics and principles of robotics as a means of motivation in the insertion of higher education for generation of future engineers.
\end{abstract}

Keywords: Education. Robotic. Technology. High School. 